\title{
PAUCI-IMMUNE CRESCENTIC GLOMERULONEPHRITIS IN PATIENT WITH SYSTEMIC LUPUS ERYTHEMATOSUS: CASE REPORT
}

\begin{abstract}
Caroline Coutinho Pires ${ }^{1, \star}$, Driena Costa Batista ${ }^{1}$, Paulo Geovanny Pedreira ${ }^{1}$, Danilo Garcia Ruiz' ${ }^{1}$, Allethéa Robertha Souza e Silva ${ }^{1}$, Daniela Maria Edilma Japiassu Custodio ${ }^{1}$, Paola Bottin Madrid ${ }^{1}$, Rafaela Alen Costa Freire ${ }^{1}$, Leticia Lopes Coimbra ${ }^{1}$
\end{abstract}

1. Universidade Federal do Tocantins, Palmas (TO), Brazil.

*Corresponding author: caroline_cpires@hotmail.com

\section{BACKGROUND}

Rapidly progressive glomerulonephritis is divided into three subtypes: type I, associated with anti-glomerular basement membrane antibodies; type II, mediated by immune complexes and associated with diseases such as systemic lupus erythematosus (SLE) and IgA nephropathy; and type III, pauci-immune, with few deposits of immunoglobulin, characteristic of ANCA-associated vasculitis. The association between pauci-immune crescentic glomerulonephritis (PICGN) and SLE is rare, described in only a few case reports.

\section{CASE REPORT}

A 17-year-old female with a previous diagnosis of SLE for 5 years. At the time, she presented with generalized edema, polyarthralgia, pleural and pericardial effusion, oral ulcers and nephritis ( $7 \mathrm{~g} / 24 \mathrm{~h}$ ), positive antinuclear antibody (ANA), positive anti-DNA and low complement. There were no manifestations suggestive of systemic vasculitis. She was initially treated with methylprednisolone $1 \mathrm{~g} /$ day for 3 days, followed by monthly cyclophosphamide for 9 months. Renal biopsy showed class IV lupus nephritis. A new induction was performed with mycophenolate mofetil $3 \mathrm{~g} /$ day due to the refractoriness of the condition, followed by maintenance with mycophenolate at a dose of $1.5 \mathrm{~g} /$ day. Currently showing relapse of proteinuria ( $9 \mathrm{~g} / 24 \mathrm{~h}$ ), low complement, positive anti-DNA and p-ANCA-positive. Antiproteinase 3 and antimyeloperoxidase antibodies were negative. There was a worsening of kidney function with indication for hemodialysis. A new biopsy was performed, which was suggestive of crescentic glomerulonephritis, in the proliferative/sclerosing phase, pauci-immune form, which may correspond to ANCA-associated vasculitis. Based on the biopsy result, pulse therapy with methylprednisolone $1 \mathrm{~g}$ was performed for 3 days and rituximab $1 \mathrm{~g}$ was started, with a schedule of doses on days 0 and 14 every 6 months. The patient has so far evolved with stabilization of renal function, without the need for further hemodialysis sessions.

\section{CONCLUSION}

The association between SLE and pauci-immune crescentic glomerulonephritis is rare. Renal damage in this case can be secondary to SLE or due to primary vasculitis. The presence of positive ANCA may suggest an overlap with ANCA-associated vasculitis, such as granulomatosis with polyangiitis, microscopic polyangiitis, or kidney-limited vasculitis. There is no specific protocol for cases of SLE associated with PICGN, but standard treatment for class IV lupus nephritis has been used in case reports. It is common the use of the combination between corticosteroids and cyclophosphamide, or alternatively, rituximab, for refractory cases. The prognosis is variable and depends largely on the degree of renal dysfunction at the time of diagnosis.

\section{KEYWORDS}

Glomerulonephritis, Vasculitis, Lupus erythematosus, Systemic. 